\title{
The comparative population genetics of Neisseria meningitidis and Neisseria gonorrhoeae
}

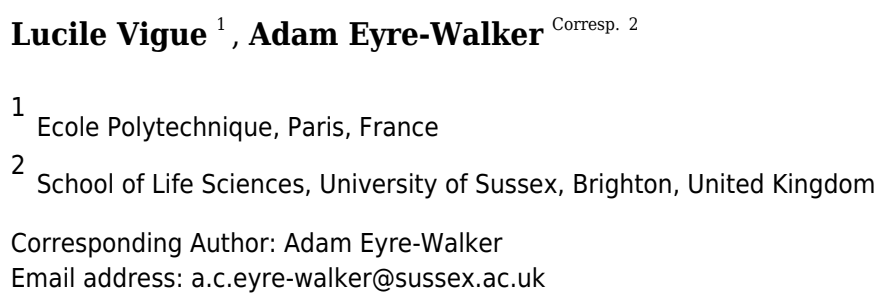

Neisseria meningitidis $(\mathrm{Nm})$ and $\mathrm{N}$. gonorrhoeae $(\mathrm{Ng})$ are closely related pathogenic bacteria. To compare their population genetics we compiled a dataset of 1145 genes found across $20 \mathrm{Nm}$ and $15 \mathrm{Ng}$ genomes. We find that $\mathrm{Nm}$ is seven-times more diverse than $\mathrm{Ng}$ in their combined core genome. Both species have acquired the majority of their diversity by recombination with divergent strains, however we find that $\mathrm{Nm}$ has acquired more of its diversity by recombination than $\mathrm{Ng}$. We find that linkage disequilibrium declines rapidly across the genomes of both species. Several observations suggest that $\mathrm{Nm}$ has a higher effective population size than $\mathrm{Ng}$; it is more diverse, the ratio of non-synonymous to synonymous polymorphism is lower, and linkage disequilibrium declines more rapidly to a lower asymptote in $\mathrm{Nm}$. The two species share a modest amount of variation, half of which seems to have been acquired by lateral gene transfer and half from their common ancestor. We investigate whether diversity varies across the genome of each species and find that it does. Much of this variation is due to different levels of lateral gene transfer. However, we also find some evidence that the effective population size varies across the genome. We test for adaptive evolution in the core genome using a McDonald-Kreitman test and by considering the diversity around non-synonymous sites that are fixed for different alleles in the two species. We find some evidence for adaptive evolution using both approaches. 
1 The comparative population genetics of Neisseria meningitidis and Neisseria gonorrhoeae

2

3

4

5

6

7

\section{Abstract}

\author{
Lucile Vigue $^{1}$ \\ Adam Eyre-Walker ${ }^{2}$
}

1. Ecole Polytechnique

Paris

France

2. School of Life Sciences

University of Sussex

Brighton

United Kingdom

Correspondence : a.c.eyre-walker@sussex.ac.uk

Neisseria meningitidis $(\mathrm{Nm})$ and $\mathrm{N}$. gonorrhoeae $(\mathrm{Ng})$ are closely related pathogenic bacteria. To compare their population genetics we compiled a dataset of 1145 genes found across $20 \mathrm{Nm}$ and $15 \mathrm{Ng}$ genomes. We find that $\mathrm{Nm}$ is seven-times more diverse than $\mathrm{Ng}$ in their combined core genome. Both species have acquired the majority of their diversity by recombination with divergent strains, however we find that $\mathrm{Nm}$ has acquired more of its diversity by recombination than $\mathrm{Ng}$. We find that linkage disequilibrium declines rapidly across the genomes of both species. Several observations suggest that $\mathrm{Nm}$ has a higher effective population size than $\mathrm{Ng}$; it is more diverse, the ratio of non-synonymous to synonymous polymorphism is lower, and linkage disequilibrium declines more rapidly to a lower asymptote in $\mathrm{Nm}$. The two species share a modest amount of variation, half of which seems to have been acquired by lateral gene transfer and half from their common ancestor. We investigate whether diversity varies across the genome of each species and find that it does. Much of this variation is due to different 
30 levels of lateral gene transfer. However, we also find some evidence that the effective population size varies across the genome. We test for adaptive evolution in the core genome using a McDonald-Kreitman test and by considering the diversity around non-synonymous sites that are fixed for different alleles in the two species. We find some evidence for adaptive evolution using both approaches.

\section{Introduction}

The two closely related bacteria Neisseria meningitidis $(\mathrm{Nm})$ and Neisseria gonorhoeae $(\mathrm{Ng})$ are major human pathogens. $\mathrm{Ng}$ is the causative agent of the sexually transmitted disease gonorrhoeae which currently infects 106 million people each year worldwide (WHO 2012). When untreated, gonoccocal infections can result in long-term problems such as persistent urethritis, cervicitis, proctitis, pelvic inflammatory disease, infertility, first-trimester abortion, ectopic pregnancy and maternal death (WHO 2012). They also increase the risk of acquiring and transmitting HIV. In cases of pregnancy, $\mathrm{Ng}$ infections can cause severe damages to neonatal health (WHO 2012). In contrast, $\mathrm{Nm}$ is a human commensal infecting approximately $10 \%$ of the healthy human population (Claus et al. 2005; Yazdankhah et al. 2004), which only occasionally causes disease. However, it can cause meningococcal meningitidis and septicaemia with mortality rates that can reach $50 \%$ when untreated, and the global disease burden is estimated to be $\sim 500,000$ cases a year (Roberts 2008). Among the different micro-organisms that can cause meningitidis, it is regarded as one of the most important because of its ability to cause large epidemics.

Here we consider several aspects of the population genetics of these bacterial species. The two species are sister taxa (Bennett et al. 2012), and Nm is known to be considerably more diverse than Ng within the genes that they share in common (Bennett et al. 2012; Bennett et al. 2007). The first problem we address is why the two taxa differ in their diversities. There are several potential explanations. First, Ng might have a lower effective population size, either because it evolved from $\mathrm{Nm}$ and went through a bottleneck when the species was formed (Vazquez et al. 
1993), or because it generally has a lower effective population size, possibly because it has a lower census population size. Second, $\mathrm{Ng}$ might have a lower mutation rate than $\mathrm{Nm}$. Third, $\mathrm{Ng}$ might acquire less diversity through recombination than $\mathrm{Nm}$. Both $\mathrm{Ng}$ and $\mathrm{Nm}$ are known to be naturally transformable, and it has been known for many years that both species acquire diversity, within their core genome, by homologous recombination with genetically divergent strains (Spratt 1988; Spratt et al. 1989). We refer to this process as homologous lateral gene transfer (hLGT), to differentiate it from the acquisition of accessory genes by non-homologous lateral gene transfer (nhLGT) (however, note that the acquisition of new genes generally involves homologous recombination with flanking genes, so nhLGT will typically involve some hLGT (Kong et al. 2013)). hLGT leads to mosaic genes, in which parts of the gene have been acquired from a highly divergent strain or a different bacterial species. In fact, $\mathrm{Nm}$ and $\mathrm{Ng}$ were some of the first bacteria in which this form of recombination was demonstrated (Spratt 1988; Spratt et al. 1989). It has been estimated that $\mathrm{Nm}$ acquires single nucleotide polymorphisms (SNPs) through hLGT at a rate between 4 and 100x higher than via mutation (Feil et al. 2001; Hao et al. 2011; Kong et al. 2013; Vos \& Didelot 2009). In contrast this ratio has recently been estimated to be only about two-fold in $\mathrm{Ng}$ (Ezewudo et al. 2015). It is unclear whether these ratios are significantly different. We investigate this here.

The second question, we address is whether diversity varies across the core genome of the two species. Genetic diversity is known to vary across the genome of many species. This was originally demonstrated in Drosophila melanogaster by Begun and Aquadro (Begun \& Aquadro 1992) who showed that diversity was positively correlated to the rate of recombination. This was thought to be due to the effects of linked selection, in the form of genetic hitch-hiking (Maynard Smith \& Haigh 1974) and background selection (Charlesworth et al. 1993), depressing diversity in regions of the genome with low rates of recombination. Variation in diversity across the genome has been demonstrated in many other species including the bacterium Escherichia coli (Maddamsetti et al. 2015; Martincorena et al. 2012). The reasons for this variation remain unclear (Chen \& Zhang 2013; Maddamsetti et al. 2015; Martincorena \& Luscombe 2013). 
88 The final question we address is whether $\mathrm{Nm}$ and $\mathrm{Ng}$ have undergone adaptive evolution. $\mathrm{Nm}$ 89 and $\mathrm{Ng}$ inhabit different niches and one presumes they have undergone adaptive evolution to 90 allow them to do this. Some of this adaptation may have been through the acquisition of new 91 genes via nhLGT, but there might also be adaptation in the core genome. Two recent analyses 92 using the $d_{N} / d_{S}$ test on the core genome have found limited evidence for adaptive evolution in $93 \mathrm{Nm}$ (Yu et al. 2014) and $\mathrm{Ng}$ (Ezewudo et al. 2015), but this test is known to be very 94 conservative. Here we apply two additional tests. 
95 Materials and methods

96 Dataset

97 All 15 genomes of Neisseria gonorrhoeae that were present in Genbank in April 2018

98 (NCCP11945 (Chung et al. 2008), FA19 (Abrams et al. 2015), FA6140 (Abrams et al. 2015), 35/02

99 (Abrams et al. 2015), FA 1090, MS11, FA19, FA6140, 35/02, 32867, 34530, 34769, FDAARGOS

100 204, FDAARGOS 205, FDAARGOS 207, NCTC13799, NCTC13798, NCTC13800) and 20 randomly

101 selected genomes of Neisseria meningitidis (MC58 (Tettelin et al. 2000), Z2491 (Parkhill et al.

102 2000), FAM18 (Bentley et al. 2007), 053442 (Peng et al. 2008), alpha14 (Schoen et al. 2008),

1038013 (Rusniok et al. 2009), alpha710 (Joseph et al. 2010), WUE 2594 (Schoen et al. 2011),

104 G2136 (Budroni et al. 2011), M01-240149 (Budroni et al. 2011), M04-240196 (Budroni et al.

105 2011), H44/76 (Budroni et al. 2011), M01-240355 (Budroni et al. 2011), NZ-05/33 (Budroni et al.

106 2011), 510612 (Zhang et al. 2014), NM3686, M7124, NM3682, NM3683, L91543) were

107 downloaded from Genbank. From these all protein coding sequences were extracted. We

108 retained those coding sequences that started NTG, terminated with TAA, TAG or TGA and had a

109 length that is a multiple of three. We identified orthologs using reciprocal BLAST, with an e-

110 value threshold of 0.00001 ; i.e. each protein coding gene in each genome was BLASTed against

111 the genes of FA1090, and then the best hit was BLASTed back onto the original genome,

112 retaining only those hits in which the original query sequence was the best hit. Similar

113 selections of genes were obtained using alternative starting genomes. The protein sequences of

114 the orthologs were aligned using MUSCLE (Edgar 2004). We selected genes where the

115 alignments meet these criteria: the number of gaps is lower than $1 \%$ of the length of the

116 sequence and the total number of nucleotides in gaps is lower than $10 \%$ of the total number of

117 nucleotides in the sequence. Sequences with internal stop codons were removed. This resulted

118 in a dataset of 1145 genes belonging to the core genome of both Neisseria gonorrhoeae and

119 Neisseria meningitidis. We used the BioPython Phylo library (Cock et al. 2009) to estimate a

120 phylogeny of the strains based on the core genome alignment.

121

122 Analyses

Peer] reviewing PDF | (2018:11:32791:2:1:NEW 23 May 2019) 
123 In most analyses we treated genes independently. However, to detect hLGT we ran

124 ClonalFrameML (Didelot \& Wilson 2015) on a concatenation of the protein coding sequences

125 from the core genome of both species. Genes were concatenated randomly without respect for

126 synteny. For some analyses we masked those regions inferred to be due to hLGT in the strains

127 affected.

128

129 We investigated whether linkage disequilibrium (LD) declines with the distance between sites

130 by measuring the LD between all pairs of polymorphisms within each gene; we did not

131 concatenate the genes or align whole genomes, because with the gain and loss of genes the

132 distance between sites differs depending on the strains being analysed. We measured LD using

133 the $r^{2}$ statistic (Hill \& Robertson 1968). LD values were then assigned to bins based on the

134 distance between the two sites - 10bp bins between 1-100bp, a bin from 101-200bp and then

135 200bp bins between 201-800bp. We took the average LD and distance between sites for each

136 bin in a manner which weighted each gene equally - we estimated the average LD and distance

137 for pairs of sites in each bin for each gene and then averaged those values across genes. To

138 estimate the approximate half-life of LD, we found the distance between sites that gave

139 approximately half the LD between the LD for the 1-10bp bin and the asymptotic value of the

140 LD.

141

142 Because $r^{2}$ is constrained to be positive, the expected value of $r^{2}$ is greater than zero even when

143 there is no LD. To calculate the expected value of $r^{2}$ when there is no LD, we considered two bi-

144 allelic loci with alleles at frequencies $p_{1}$ and $p_{2}$. The expected frequencies of the four haplotypes

145 are $p_{1} p_{2}, p_{1}\left(1-p_{2}\right)$...etc. from which we generated four random variates from a multinomial

146 distribution for a sample size of $N$ chromosomes using Mathematica version 11; for each

147 sample of haplotypes we calculated $r^{2}$. We repeated this procedure 10,000 times and calculated

148 the mean to estimate the expected value of $r^{2}$. We found that the expected value of $r^{2}$ is

149 independent of the allele frequencies.

150

Peer] reviewing PDF | (2018:11:32791:2:1:NEW 23 May 2019) 
151 To investigate the relationship between the non-synonymous, $\pi_{N}$, and synonymous, $\pi_{\mathrm{s}}$,

152 nucleotide diversity we used a variation of the method of James et al. (James et al. 2017) to

153 combine data from different genes. If the distribution of fitness effects of new mutations is a

154 gamma distribution (assuming most mutations are deleterious) then $\log \left(\pi_{N}\right)$ is expected to be

155 linearly correlated to $\log \left(\pi_{s}\right)$ if there is variation in $N_{e}$ (Welch et al. 2008). However, for many

156 genes either $\pi_{N}$ or $\pi_{S}$ is zero, hence we need to combine genes together. We can do this by

157 splitting the synonymous polymorphisms into two groups according to whether they were in an

158 odd or even numbered codon and then using the two groups to estimate two synonymous

159 nucleotide diversities that have independent sampling errors, $\pi_{\mathrm{S} 1}$ and $\pi_{\mathrm{S} 2}$. One of these, $\pi_{\mathrm{S} 1}$,

160 was used to rank and group genes, and the other was averaged across genes in the group to

161 give an unbiased estimate of $\pi_{S}$ for the group. $\pi_{N}$ was also averaged across the genes in the

162 group.

163

164 To investigate the diversity around sites that are fixed between $\mathrm{Nm}$ and $\mathrm{Ng}$ for different alleles

165 we focused on genes that had at least one synonymous polymorphism and one fixed difference

166 between the two species. For each fixed difference, we identified all the synonymous

167 polymorphisms that were within $1 \mathrm{~kb}$ and we grouped them by windows of $100 \mathrm{bp}$. Since,

168 background selection can potentially lead to a lower dip in diversity around fixed non-

169 synonymous mutations we normalised the diversity around fixed synonymous and non-

170 synonymous substitutions by dividing the number of synonymous polymorphisms in a particular

171 window by the total number of synonymous polymorphisms in the gene, multiplied by the

172 window size over the gene length.

173

174

175 Results

176 Recombination and mutation

177 We are interested in how genetic variation is generated and distributed in the two Neisseria

178 species $\mathrm{N}$. meningitidis $(\mathrm{Nm})$ and $\mathrm{N}$. gonorrhoeae $(\mathrm{Ng})$. Although, the presence and absence of

179 genes in the strains of the two species is an important aspect of this problem, here we focus on 
180 the genetic variation that is present in the core genome that is common to both species. Using

181 reciprocal BLAST, we identified 1145 genes present across the 15 genomes of $\mathrm{Ng}$ and 20

182 genomes of $\mathrm{Nm}$ that we analysed. The total length of this core genome is 1.1MB long. Defining

183 a polymorphism as a site that contains two or more alleles within either of the two species, we

184 find that $\mathrm{Nm}$ is $\sim 7.6$ fold more diverse than $\mathrm{Ng}$ consistent with previous qualitative reports

185 (Bennett et al. 2012; Bennett et al. 2007). The difference in diversity is more apparent at

186 synonymous ( $~ 8.9$ fold) than non-synonymous ( 5.5 fold) sites (Table 1 ), a pattern we return to

187 later. The two species share a modest amount of diversity; $35 \%$ of all polymorphisms in $\mathrm{Ng}$ are

188 shared with $\mathrm{Nm}$, and $4.5 \%$ of those in $\mathrm{Nm}$ are shared with $\mathrm{Ng}$.

189

190 It is well known that $\mathrm{Nm}$ and $\mathrm{Ng}$ undergo substantial levels of homologous recombination with

191 divergent strains, possibly from other species of bacteria. This leads both to the acquisition of

192 new genes, but also to the acquisition of parts of genes that are already present in the genome;

193 we refer to these processes as non-homologous (nhLGT) and homologous lateral gene transfer

194 (hLGT) respectively. To quantify the role that hLGT plays in the acquisition of diversity in the

195 core genome we ran ClonalFrameML (Didelot \& Wilson 2015) (Didelot \& Wilson 2015). The

196 method estimates the ratio of the rate at which recombination tracts initiate $(R)$ and the rate of

197 mutation $(\theta)$, both multiplied by twice the effective population size, $N_{e}$, along with the average

198 recombination tract length, $\delta$, and the proportion of sites that differ between the imported and

199 resident sequences, $v$. Estimates of these parameters are given in Table 2 . The overall effect of

200 recombination relative to mutation can be estimated as $R \delta v / \theta=r / m$, where $r$ and $m$ are the

201 rates at which variants are introduced into a genome by recombination and mutation

202 respectively.

203

204 In Nm we find that recombination introduces $6.43(95 \% \mathrm{Cl}=6.16$ to 6.71$)$ times more variation

205 than mutation, whereas in $\mathrm{Ng}$ it introduces $1.97(1.76,2.19)$ times as much. In $\mathrm{Nm}$ the $\mathrm{r} / \mathrm{m}$ ratio

206 has previously been estimated to be 5.37(Hao et al. 2011), 6.71 (Vos \& Didelot 2009), 16.4

207 (Kong et al. 2013) and 100 (Feil et al. 2001). Our estimate is similar to the first two estimates,

208 but substantially lower than the last two estimates. Both of these latter estimates were 
obtained from very closely related strains and hence may reflect the value of $\mathrm{r} / \mathrm{m}$ before natural selection has had an opportunity to operate. In $\mathrm{Ng}$ it has been estimated that $2.2 \mathrm{x}$ as

211 much variation is introduced by recombination (Ezewudo et al. 2015), which is very similar to

212 our estimate. The estimates of $\mathrm{r} / \mathrm{m}$ mean that $\sim 87 \%$ of all polymorphisms in $\mathrm{Nm}$ are a

213 consequence of recombination, whereas in $\mathrm{Ng}$ it is $66 \%$. The difference between the two

214 species in the influence of recombination is largely driven by a difference in the ratio of the rate

215 at which recombination is initiated versus the mutation rate $(R / \theta)$, since although the tract

216 lengths are estimated to be on average slightly longer in $\mathrm{Nm}$, they introduce slightly less

217 variation than $\mathrm{Ng}$ (Table 2).

218

219 ClonalframeML estimates the ratio of $R$ and $\theta$ but not their absolute values. However, we can

220 estimate the absolute value as follows. We note that the nucleotide diversity is due to the input of mutation and the input of recombination: i.e. $\pi=\theta+R \delta$ v. If we note that ClonalframeML gives us an estimate of $R / \theta$ we can rewrite this equation as $\pi=\theta+\theta \delta \cup R / \theta$, from which we can estimate $\theta=\pi /(1+\delta \cup \mathrm{R} / \theta)$. Estimates of $R$ and $\theta$ are given in Table 2 . From this it is evident that the nucleotide diversity is higher in $\mathrm{Nm}$ both because of a 3-fold greater mutational input and a nine-fold greater rate at which recombination tracts initiate in $\mathrm{Nm}$, at the population level (i.e. when the tract length initiation rate and mutation rate are multiplied by $N_{e}$ ).

The parameters $R$ and $\theta$ are the rates of recombination initiation and mutation, multiplied by the effective population size. Hence a simple reason why both parameters are higher in $\mathrm{Nm}$ might simply be that $\mathrm{Nm}$ has a higher $N_{e}$ than $\mathrm{Ng}$. To test this idea, we masked all sequences that were identified as due to hLGT by ClonalframeML and estimated the levels of nonsynonymous and synonymous diversity. Under a model in which synonymous mutations are neutral and non-synonymous mutations are deleterious, but drawn from some distribution, we

234 expect $\pi_{\mathrm{N}} / \pi_{\mathrm{S}}$ to be lower in species with high $N_{e}$; this is because selection is more effective in 235 species with higher $N_{e}$ and hence the proportion of mutations that are effectively neutral is 236 lower (Ohta 1972; Ohta 1977; Ohta 1992). This is what we find $-\pi_{N} / \pi_{S}=0.095(S E=0.0023)$ in 
$237 \mathrm{Nm}$ versus $0.23(0.014)$ in $\mathrm{Ng}$. These are significantly different to each other (normal test $\mathrm{z}=9.5$, $238 \mathrm{p}<0.001)$.

239

240 As we described above, $\mathrm{Nm}$ and $\mathrm{Ng}$ share a modest amount of genetic variation. It is of some

241 interest whether this is a consequence of hLGT or the inheritance of genetic variation from

242 their common ancestor. If we exclude those sequences inferred to be due to hLGT we find that

243 the two species still share a modest amount of genetic variation $-15.5 \%$ of all $\mathrm{Ng}$

244 polymorphisms are shared with $\mathrm{Nm}$ and $2.4 \%$ of $\mathrm{Nm}$ polymorphisms are shared with $\mathrm{Ng}$,

245 approximately half of all shared polymorphisms in each case, suggesting that some proportion

246 of the shared variation originated from their common ancestor.

248 Linkage disequilibrium

249 Homologous recombination can both increase and decrease linkage disequilibrium (LD);

250 homologous recombination with divergent strains, of the sort detected by ClonalFrameML,

251 generates LD because it simultaneously introduces many polymorphisms that are initially linked

252 to each other. However, homologous recombination amongst a set of closely related strains

253 breaks-up LD. To investigate how these two forces play out, we calculated the LD between all

254 pairs of sites within each gene and plotted these as a function of the distance between sites. As

255 expected we observe a decline in LD with distance (Figure 1A). Both species show similar

256 patterns with LD declining rapidly; in Nm the approximate half-life is 30bp and in Ng it is $100 \mathrm{bp}$.

257 The decline could be due to two processes. If most hLGT fragments tend to be short, with

258 decreasing numbers of long fragments, then LD will be greater between closely linked sites.

259 However, we also expect a decline due to recombination between closely related strains, and in

260 fact we observe a decline even when we focus on those parts of the genome which do not

261 appear to have undergone hLGT (Figure 1B).

262

263 In both species LD asymptotes above zero. The non-zero asymptote could be due to one of

264 three reasons - statistical bias, population substructure and a balance between genetic drift

265 and recombination. The statistical bias arises because our measure of LD, $r^{2}$ (Hill \& Robertson 
266 1968), cannot be negative, so positive values of $r^{2}$ are expected even if there is no LD if sample 267 sizes are small; for sample sizes of 15 and 20 strains, the expected value of $r^{2}$ is 0.079 and 0.050

268 respectively (see materials and methods), so the asymptote is clearly above this level. Both, Nm 269 and $\mathrm{Ng}$ have been shown to have some level of population structure so this is the likely to be 270 part of the explanation (Budroni et al. 2011; Joseph et al. 2011). However, the slower decay in

$271 \mathrm{LD}$, and higher asymptote in $\mathrm{Ng}$, is consistent with $\mathrm{Ng}$ having a smaller $\mathrm{N}_{e}$ than $\mathrm{Nm}-$ i.e. the 272 non-zero asymptote might in part be caused by a balance between genetic drift creating LD, 273 and recombination breaking it down.

274

275

Diversity across the genome

276 Nucleotide diversity is known to vary across the genomes of many organisms. This is largely

277 thought to be driven by variation in the mutation rate or variation in the effects of linked

278 selection. However, in bacteria, and particularly $\mathrm{Nm}$ and $\mathrm{Ng}$, it could also be due to variation in

279 the frequency of hLGT. All of these processes are expected to affect synonymous and non-

280 synonymous diversity to greater or lesser extents, and indeed we observe a positive correlation

281 between non-synonymous and synonymous diversity, demonstrating that both vary across the

282 genome in concert. At least part of this pattern is driven by hLGT because genes with hLGT

283 show higher $\pi_{N}$ and $\pi_{S}$ values than genes without any evidence of hLGT (Figure 2 ).

285 However, to investigate whether there is also variation in the effective population size across 286 the genome we removed sequences inferred to be due to hLGT by ClonalFrameML from our 287 data. This reduces our data substantially and so to reduce statistical sampling issues we used 288 the method of James et al. (2016) to combine data from different genes. We find that $\pi_{\mathrm{N}}$ and $\pi_{\mathrm{S}}$ 289 are still significantly correlated suggesting the correlation between them is not just driven by $290 \mathrm{hLGT}(\mathrm{Ng}$ slope $=0.23, \mathrm{p}<0.001 ; \mathrm{Nm}$ slope $=0.53, \mathrm{p}<0.001)$ (Figure 3). The remaining correlation 291 could be due to variation in the mutation rate or variation in the effects of linked selection. We 292 can test whether there is variation in the effects of linked selection by considering the slope 293 between $\log \left(\pi_{N}\right)$ and $\log \left(\pi_{S}\right)$. Under a model in which there is no variation in linked selection 294 then the slope of this relationship is expected to be one, and if there is variation in linked 
295 selection the slope if expected to be less than one (Galtier 2016; Welch et al. 2008). Linked 296 selection has two consequences. First, it increases the stochasticity in allele frequencies. For

297 example, the spread of an advantageous mutation or the elimination of deleterious genetic 298 variation, removes linked genetic diversity; whether a linked mutation survives either process is

299 a random process depending on whether the advantageous or deleterious mutation occurs in

300 linkage with the target mutation. This can be thought of as reduction in the effective population

301 size. Second, genetic hitch-hiking leads to non-equilibrium dynamics. After a selective sweep,

302 genetic diversity will recover, but this happens faster for deleterious than neutral mutations

303 (Brandvain \& Wright 2016; Do et al. 2015; Gordo \& Dionisio 2005). In both cases we expect a

304 negative correlation between $\pi_{\mathrm{N}} / \pi_{\mathrm{S}}$ and $\pi_{\mathrm{S}}$, which manifests itself in a positive correlation

305 between $\log \left(\pi_{N}\right)$ and $\log \left(\pi_{S}\right)$ but with a slope of less than one (James et al. 2017). We find that

306 the slope of the relationship between $\log \left(\pi_{N}\right)$ and $\log \left(\pi_{S}\right)$ is $0.23(\mathrm{SE}=0.052)$ and $0.59(0.070)$

307 for $\mathrm{Ng}$ and $\mathrm{Nm}$ respectively, in both cases significantly less than one $(p<0.001)$; i.e. $\pi_{\mathrm{N}}$ increases

308 as $\pi_{\mathrm{s}}$ increases but not as fast. The slopes are significantly different to each other (t-test,

$309 \mathrm{p}<0.001)$.

310

311 Adaptive evolution

$312 \mathrm{Nm}$ and $\mathrm{Ng}$ are ecologically quite different and one presumes the two species have undergone

313 adaptation to live in their respective environments. Some of this adaptation will have come

314 about through the acquisition of whole genes through nhLGT. However, some of the adaptation

315 may have occurred within the core genome of the two species either by new mutations,

316 standing genetic variation, or hLGT. To investigate whether there has been adaptation in the

317 core genome we used two approaches. First, we used the McDonald-Kreitman (McDonald \&

318 Kreitman 1991) approach to estimate the rate of adaptive evolution (Eyre-Walker 2006; Fay et

319 al. 2001). In this method the numbers of non-synonymous and synonymous substitutions (i.e.

320 differences between the two species, $d_{N}$ and $d_{S}$ respectively) are compared to the numbers of

321 non-synonymous and synonymous polymorphisms ( $p_{N}$ and $p_{S}$ respectively). Under a neutral

322 model in which mutations are either neutral or strongly deleterious we expect $d_{N} / d_{S}=p_{N} / p_{S}$

323 (McDonald \& Kreitman 1991). In contrast if there are slightly deleterious non-synonymous 
324 mutations we expect $d_{N} / d_{S}<p_{N} / p_{S}$, and if there are some advantageous mutations we expect $325 d_{N} / d_{S}>p_{N} / p_{S}$ (Eyre-Walker 2006; Fay et al. 2001). Summing $d_{N}, d_{S}, p_{N}$ and $p_{S}$ we calculate the

326 fixation index $F I=d_{N} p_{S} / d_{S} p_{N}$ (Gojobori et al. 2007); adaptive evolution is indicated if $F I>1$.

328 We find that our estimate of $\mathrm{Fl}$ differs if we use the polymorphism data of $\mathrm{Nm}$ or $\mathrm{Ng}$; using the 329 SNP data of $\mathrm{Nm}$ we estimate that $F /$ is significantly greater than one suggesting adaptive 330 evolution has occurred ( $F I=1.51$ with $95 \%$ Cis $=1.41$ and 1.61$)$, but if we use the SNP data of $331 \mathrm{Ng}$, our estimate is significantly less than one $(F I=0.92(0.83,0.99))$. Estimates less than one 332 can occur if there are slightly deleterious mutations (SDMs) segregating, but even if we restrict 333 our analysis to common polymorphisms, which should remove many of the SDMs

334 (Charlesworth \& Eyre-Walker 2008; Fay et al. 2001), we find that the $F /<1$ using the SNP data of $335 \mathrm{Ng}$ (using SNPs with allele frequencies above $15 \%, F I=0.78(0.78,0.88)$ ). An explanation for 336 why $\mathrm{Fl}$ differs between the two species is that either $\mathrm{Nm}$ has undergone population expansion, 337 or Ng has undergone contraction. If there are slightly deleterious mutations then population 338 size expansion leads to an overestimate of $F /$ whereas contraction leads to an underestimate 339 (Eyre-Walker 2002; McDonald \& Kreitman 1991). As we argue above, a simple explanation for 340 why $\mathrm{Nm}$ is more diverse than $\mathrm{Ng}$ is that $\mathrm{Nm}$ has a higher $\mathrm{N}_{e}$. We find no evidence of expansion or contraction amongst the current strains - Tajima's D (Tajima 1989), a measure of a skew in

342 the site frequency spectrum away from what we expect for neutral mutations in a stationary

343 population size is close to zero and not significantly different to zero in both species in the

344 regions of the genome that have no evidence of hLGT (Tajima's D $=-0.073$ and -0.093 in Nm and

$345 \mathrm{Ng}$ respectively), consistent with previous analyses in $\mathrm{Nm}$ (Joseph et al. 2011). However, the 346 expansion or contraction in either $\mathrm{Nm}$ or $\mathrm{Ng}$ could have occurred sometime in the past which

347 would not be visible to an analysis using Tajima's D, but which might still affect the FI.

348

349 A second approach to test for adaptive evolution, is to investigate whether there is a dip in 350 genetic diversity around putatively advantageous mutations (Sattath et al. 2011) - as

351 advantageous mutations spread through a population it reduces diversity in its proximity. We

352 find that synonymous diversity is lower close to sites that are fixed for different nucleotides in 
353 the two species, and this dip is significantly greater for non-synonymous than synonymous fixed

354 differences when considering diversity in $\mathrm{Nm}$ ( $\mathrm{p}<0.001$ for distances 1-100bp, 101-200bp and

355 201-300bp), consistent with a proportion of non-synonymous mutations being fixed by positive

356 adaptive evolution; a similar pattern is not evident in $\mathrm{Ng}$, possibly because it is less diverse.

358 There is however an alternative explanation for the greater dip around non-synonymous

359 substitutions; if the strength of background selection (Charlesworth et al. 1995) varies across

360 the genome, then regions with high levels of background selection will have low diversity but

361 will tend to also fix slightly deleterious non-synonymous mutations. To investigate whether

362 there is evidence of this, we considered whether the ratio of the non-synonymous to

363 synonymous substitution rates, $\log \left(d_{N} / d_{s}\right)$, was correlated to synonymous diversity, $\log \left(\pi_{s}\right)$. We

364 again use the method of James et al. (James et al. 2017) to combine data from different genes

365 and find that a strong negative correlation in $\mathrm{Nm}$ (slope $=-0.10, \mathrm{p}=0.018$ ) but not in $\mathrm{Ng}$ (slope

$366=-0.001, p=0.97)$. This suggests that background selection might be a factor in $\mathrm{Nm}$. To take into

367 account the potential variation in background selection, we normalised the data from each

368 gene by dividing the number of synonymous SNPs in each window by the average diversity in

369 each gene. This will account for variation in background selection at a gene level, but not at a

370 sub-gene level. The normalised data show a greater dip in diversity for fixed non-synonymous

371 than synonymous substitutions in both $\mathrm{Nm}$ (combining t-test results from the three closest

372 points, $p<0.001)$, and $\mathrm{Ng}(\mathrm{p}=0.0024)$ (figure 4) although the differences are not large.

373

\section{Discussion}

375 We have investigated several aspects of the comparative population genetics of the two

376 bacteria Neisseria meningitidis and N. gonorrhoeae. We find, as others have (Bennett et al.

377 2012; Bennett et al. 2007), that $\mathrm{Nm}$ is substantially more diverse than $\mathrm{Ng}$, but that the two

378 species share a moderate amount of diversity in the genes that they have in common. This

379 shared diversity could have been a consequence of ancestral polymorphism that has been

380 inherited by both species, or due to hLGT transferring variation between the two. We find a

381 substantial fraction is indeed due to $\mathrm{hLGT}$, since if we remove the fraction of the genome that 
382 appears to have undergone hLGT, the fraction of shared polymorphism drops considerably.

383 However, there is some diversity that appears to have been inherited from the ancestor.

384

385 In both species we find that most of their genetic diversity has been acquired by recombination,

386 rather than by mutation. In Nm we estimate that the total input from hLGT is six-fold greater

387 than from mutation; this is in line with the estimates of Hao et al. (Hao et al. 2011) and Vos and

388 Didelot (Vos \& Didelot 2009), but lower than two other estimates (Feil et al. 2001; Kong et al.

389 2013). Both of these high estimates were derived by considering very closely related strains. If

390 hLGT events are on average more deleterious than single nucleotide changes then we expect

$391 \mathrm{r} / \mathrm{m}$ estimates to be greater for more closely related strains, because natural selection has had

392 more opportunity to remove the deleterious mutations in distantly related strains. This has the

393 implication that $r / m$ may be far higher amongst newly arising mutations than often thought. In

$394 \mathrm{Ng}$ we find the input of hLGT is two-fold greater than mutation, consistent with the one

395 previous estimate performed on a similar selection of strains (Ezewudo et al. 2015).

396

$397 \mathrm{Nm}$ might be more diverse than $\mathrm{Ng}$ either $\mathrm{Nm}$ has a higher mutation rate, a greater rate of

398 hLGT or a higher effective population size. Several lines of evidence suggest that Nm has a

399 higher $N_{e}$. First, $\mathrm{Nm}$ has higher values of both $R$ and $\theta$, where $R$ and $\theta$ are estimates of the rate

400 at which recombination initiates and the mutation rate, multiplied by $N_{e}$. Second, $p_{N} / p_{S}$ is

401 lower in $\mathrm{Nm}$ in the fraction of the genome which does not seem to have undergone hLGT.

402 Third, LD declines faster in $\mathrm{Nm}$ and asymptotes at a lower level. However, this does not

403 preclude a role for either faster rates of mutation or recombination in the greater diversity in

$404 \mathrm{Nm}$.

405

406 It is possible that the lower $N_{e}$ in $\mathrm{Ng}$ is due to a bottleneck at the time when $\mathrm{Ng}$ was formed,

407 assuming that it is a derivative of $\mathrm{Nm}$ (Vazquez et al. 1993). Alternatively, it may be due to the

408 fact that $\mathrm{Ng}$ has a lower census population size. Currently $10 \%$ of the human population is

409 asymptomatically infected with Nm (Claus et al. 2005; Yazdankhah et al. 2004), whereas levels

410 of $\mathrm{Ng}$ infection are thought to be very low - between 1 and 170 cases per 100,000 individuals in 
411 Western Europe and America in 2017 (www.cdc.gov, ecdec.europa.eu). Hence, although there

412 seems to be a poor correlation between census and effective population size across species

413 (Bazin et al. 2006; Leffler et al. 2012; Lewontin 1974; Romiguier et al. 2014), we predict Nm to

414 have a much larger $N_{e}$ than $\mathrm{Ng}$, simply because it infects many more people.

415

416 In addition to the influence of hLGT we see the signature of recombination between strains of

417 the same species breaking down LD, since LD decreases with increasing distance between sites.

418 Similar patterns have been previously reported in both Nm (Budroni et al. 2011) and Ng

419 (Arnold et al. 2018) but these studies used different LD statistics and so it is hard to determine

420 what the comparative patterns are. The patterns are similar in the two species, but they are

421 consistent with a difference in $N_{e}$ since the decay in LD is faster in $\mathrm{Nm}$ and asymptotes at a

422 slightly lower value. In both species the asymptote is above what is expected under free

423 recombination even taking into account sampling error and the fact that $r^{2}$ cannot be negative

424 (see above). The asymptote might be above this level for two reasons. First, there might be a

425 balance between drift and recombination. In a gene conversion model of recombination, a non-

426 zero asymptote is expected because once sites are further apart than the gene conversion tract

427 length, then increasing distance does not increase the rate of recombination. The asymptote is

428 then determined by a balance between drift increasing LD, and recombination breaking it

429 down. The second explanation is that there is population sub-structure in both species. It has

430 been argued, based on the phylogeny of strains that there is substructure in Nm (Budroni et al.

431 2011; Kong et al. 2013) and Ng (De Silva et al. 2016; Ezewudo et al. 2015; Grad et al. 2016; Lee

432 et al. 2018). In Nm it has been suggested that this structure arises because different sets of

433 strains have different restriction modification systems (Budroni et al. 2011). However, the

434 correspondence between clades of strains and these systems is not clear cut (Kong et al. 2013).

435

436 We find as others have found in some other species, that diversity varies across the genome in

$437 \mathrm{Nm}$ and $\mathrm{Ng}$, and that this variation affects both synonymous and non-synonymous sites. This is

438 in large part driven by hLGT; regions of the genome with high rates of hLGT have high diversity.

439 However, when we focus on the part of the genome that is inferred not to have undergone 
440 hLGT we find that levels of non-synonymous and synonymous diversity are correlated, but in a 441 manner which demonstrates that $\pi_{\mathrm{N}} / \pi_{\mathrm{S}}$ declines with increasing $\pi_{\mathrm{s}}$. A similar pattern has been 442 observed within the genomes of various eukaryotes (Castellano et al. 2018; Gossmann et al. 443 2011; Murray et al. 2017) as well as between eukaryotic species (Chen et al. 2017; Galtier 2016; 444 James et al. 2017). This pattern is consistent with an influence of linked selection on the 445 genome - regions of the genome with high levels of linked selection have low $\pi_{\mathrm{S}}$, but relatively 446 high levels of $\pi_{\mathrm{N}}$. Linked selection can influence diversity in two ways. First, both background 447 selection and genetic hitch-hiking can reduce the effective population size of a genomic region. 448 Second, hitch-hiking can lead to non-equilibrium dynamics which can affect the relative levels 449 of selected and neutral diversity; after a hitch-hiking event deleterious genetic diversity will 450 return to its equilibrium value faster than neutral diversity (Brandvain \& Wright 2016; Do et al. 451 2015; Gordo \& Dionisio 2005).

452

$453 \mathrm{Nm}$ and $\mathrm{Ng}$ occupy distinct niches and one might presume that they have undergone adaptive 454 evolution. Such adaptation might have been achieved through the acquisition of new genes, 455 and/or adaptation in their core genomes. We have tested for adaptive evolution in the core 456 genome using two approaches - a McDonald-Kreitman test in which numbers of non-

457 synonymous and synonymous substitutions are compared to numbers of non-synonymous and synonymous polymorphisms (Eyre-Walker 2006; McDonald \& Kreitman 1991). We find significant evidence of adaptation when we compare the substitution data to the polymorphism data of $\mathrm{Nm}$, but no evidence if we use the polymorphism data of $\mathrm{Ng}$. These observations are consistent with a decrease in the $N_{e}$ of $\mathrm{Ng}$ or an increase in $\mathrm{Nm}$ (Eyre-Walker 2002). The difference in $N_{e}$ is consistent with the observation of higher diversity in $\mathrm{Nm}$, lower $\pi_{N} / \pi_{S}$, more rapid decay in LD and the lower asymptote in LD. However, it is difficult to resolve whether $\mathrm{Ng}$ has undergone population size contraction or $\mathrm{Nm}$ population size expansion in the past. Finally, it is tempting to estimate the fraction of substitutions fixed by adaptive evolution as $1-1 / F I-$ see (Eyre-Walker 2006). However, the simultaneous introduction of multiple mutations by hLGT makes this estimate biased. 
469 A central assumption in our analysis is that ClonalFrameML (Didelot \& Wilson 2015) has 470 correctly identified regions of the genome that have undergone hLGT. The method identifies

471 the presence of hLGT from a clustering of mutations along an inferred clonal phylogeny; a 472 sudden burst of mutations along a branch in the phylogeny, that are spatially clustered 473 together in the genome are inferred to be due to hLGT. It will therefore be difficult for the 474 method to detect hLGT with relatively similar or short sequences. Furthermore, because we

475 have used a concatenation of protein coding sequences in our ClonalframeML analysis it may 476 be difficult to detect hLGT at the start and end of genes, because we will not have the flanking 477 sequences which provide additional support for hLGT. To investigate whether this latter effect 478 is important, we plotted the number of inferred hLGT events as a function of the distance from 479 the start or end of genes. We found that events are inferred slightly less often at the start/end 480 of genes, but the effect is not large (Figure 5).

481

482 The fact that ClonalFrameML has probably missed some hLGT events suggests that we may 483 have underestimated the input of variation from hLGT in both species - i.e. we have 484 underestimated $r / m$. However, an inability to correctly detect all hLGT events is unlikely to 485 explain the differences in the relative contribution of hLGT and mutation in the two species, since both species have been treated identically. An inability to detect hLGT may however explain why $\pi_{\mathrm{N}}$ and $\pi_{\mathrm{S}}$ are correlated even in the parts of the genome with no apparent hLGT and hence there may be little or no variation in $N_{e}$ across the genomes of $\mathrm{Nm}$ and $\mathrm{Ng}$; there is an expectation that $\pi_{\mathrm{N}} / \pi_{\mathrm{S}}$ is likely to be lower amongst hLGT fragments because the polymorphisms will be dominated by mutations that are fixed between species. Furthermore, it

491 is possible that all the variation that is shared between $\mathrm{Nm}$ and $\mathrm{Ng}$ is a consequence of hLGT 492 and we have not been able to identify all hLGT events.

493

\section{Conclusions}

495 We have investigated the diversity in $\mathrm{Nm}$ and $\mathrm{Ng}$, and shown that $\mathrm{Nm}$ is more diverse then $\mathrm{Ng}$. 496 Both species have acquired most of their variation through homologous lateral gene transfer. 497 Nm appears to have higher diversity in part due to it's higher effective population size. In both 
498

499

500

501

502

503

504

505

506

507

508

509

510

511

512

513

514

515

516

517

518

519

520

521

522

523

524

525

526

527

528

529

530

531

532

533

534

535

536

537

538

species LD decays relatively slowly as a function of the distance between sites and there is some evidence of adaptive evolution in the core genome of the two species.

\section{References}

Abrams AJ, Trees DL, and Nicholas RA. 2015. Complete Genome Sequences of Three Neisseria gonorrhoeae Laboratory Reference Strains, Determined Using PacBio Single-Molecule Real-Time Technology. Genome Announc 3. 10.1128/genomeA.01052-15

Arnold BJ, Gutmann MU, Grad YH, Sheppard SK, Corander J, Lipsitch M, and Hanage WP. 2018. Weak Epistasis May Drive Adaptation in Recombining Bacteria. Genetics 208:1247-1260. 10.1534/genetics.117.300662

Bazin E, Glemin S, and Galtier N. 2006. Population size does not influence mitochondrial genetic diversity in animals. Science 312:570-572.

Begun DJ, and Aquadro CF. 1992. Levels of naturally occuring DNA polymorphism correlate with recombination rates in D. melanogaster. Nature 356:519-520.

Bennett JS, Jolley KA, Earle SG, Corton C, Bentley SD, Parkhill J, and Maiden MC. 2012. A genomic approach to bacterial taxonomy: an examination and proposed reclassification of species within the genus Neisseria. Microbiology 158:1570-1580. 10.1099/mic.0.056077-0

Bennett JS, Jolley KA, Sparling PF, Saunders NJ, Hart CA, Feavers IM, and Maiden MC. 2007. Species status of Neisseria gonorrhoeae: evolutionary and epidemiological inferences from multilocus sequence typing. BMC Biol 5:35. 10.1186/1741-7007-5-35

Bentley SD, Vernikos GS, Snyder LA, Churcher C, Arrowsmith C, Chillingworth T, Cronin A, Davis $\mathrm{PH}$, Holroyd NE, Jagels K, Maddison M, Moule S, Rabbinowitsch E, Sharp S, Unwin L, Whitehead S, Quail MA, Achtman M, Barrell B, Saunders NJ, and Parkhill J. 2007. Meningococcal genetic variation mechanisms viewed through comparative analysis of serogroup C strain FAM18. PLoS Genet 3:e23. 10.1371/journal.pgen.0030023

Brandvain Y, and Wright SI. 2016. The Limits of Natural Selection in a Nonequilibrium World. Trends Genet 32:201-210. 10.1016/j.tig.2016.01.004

Budroni S, Siena E, Hotopp JCD, Seib KL, Serruto D, Nofroni C, Comanducci M, Riley DR, Daugherty SC, Angiuoli SV, Covacci A, Pizza M, Rappuoli R, Moxon ER, Tettelin H, and Medini D. 2011. Neisseria meningitidis is structured in clades associated with restriction modification systems that modulate homologous recombination. Proceedings of the National Academy of Sciences of the United States of America 108:4494-4499. 10.1073/pnas.1019751108

Castellano D, James J, and Eyre-Walker A. 2018. Nearly neutral evolution across the Drosophila melanogaster genome. Mol Biol Evol in press.

Charlesworth B, Morgan MT, and Charlesworth D. 1993. The effect of deleterious mutations on neutral molecular variation. Genetics 134:1289-1303.

Charlesworth D, Charlesworth B, and Morgan MT. 1995. The pattern of neutral molecular variation under the background selection model. Genetics 141:1619-1632. 
539

540

541

542

543

544

545

546

547

548

549

550

551

552

553

554

555

556

557

558

559

560

561

562

563

564

565

566

567

568

569

570

571

572

573

574

575

576

577

578

579

580

581

Charlesworth J, and Eyre-Walker A. 2008. The McDonald-Kreitman test and slightly deleterious mutations. Mol Biol Evol in press.

Chen J, Glemin S, and Lascoux M. 2017. Genetic Diversity and the Efficacy of Purifying Selection across Plant and Animal Species. Mol Biol Evol 34:1417-1428. 10.1093/molbev/msx088

Chen X, and Zhang J. 2013. No gene-specific optimization of mutation rate in Escherichia coli. Mol Biol Evol 30:1559-1562. 10.1093/molbev/mst060

Chung GT, Yoo JS, Oh HB, Lee YS, Cha SH, Kim SJ, and Yoo CK. 2008. Complete genome sequence of Neisseria gonorrhoeae NCCP11945. J Bacteriol 190:6035-6036. 10.1128/JB.00566-08

Claus H, Maiden MC, Wilson DJ, McCarthy ND, Jolley KA, Urwin R, Hessler F, Frosch M, and Vogel U. 2005. Genetic analysis of meningococci carried by children and young adults. J Infect Dis 191:1263-1271. 10.1086/428590

Cock PJA, Antao T, Chang JT, Chapman BA, Cox CJ, Dalke A, Friedberg I, Hamelryck T, Kauff F, Wilczynski B, and de Hoon MJL. 2009. Biopython: freely available Python tools for computational molecular biology and bioinformatics. Bioinformatics 25:1422-1423. 10.1093/bioinformatics/btp163

De Silva D, Peters J, Cole K, Cole MJ, Cresswell F, Dean G, Dave J, Thomas DR, Foster K, Waldram A, Wilson DJ, Didelot X, Grad YH, Crook DW, Peto TEA, Walker AS, Paul J, and Eyre DW. 2016. Whole-genome sequencing to determine transmission of Neisseria gonorrhoeae: an observational study. Lancet Infectious Diseases 16:1295-1303. 10.1016/S14733099(16)30157-8

Didelot X, and Wilson DJ. 2015. ClonalFrameML: Efficient Inference of Recombination in Whole Bacterial Genomes. Plos Computational Biology 11. ARTN e1004041

10.1371/journal.pcbi.1004041

Do R, Balick D, Li H, Adzhubei I, Sunyaev S, and Reich D. 2015. No evidence that selection has been less effective at removing deleterious mutations in Europeans than in Africans. Nat Genet 47:126-131. 10.1038/ng.3186

Edgar RC. 2004. MUSCLE: multiple sequence alignment with high accuracy and high throughput. Nucleic Acids Res 32:1792-1797. 10.1093/nar/gkh340

32/5/1792 [pii]

Eyre-Walker A. 2002. Changing effective population size and the McDonald-Kreitman test. Genetics 162:2017-2024.

Eyre-Walker A. 2006. The genomic rate of adaptive evolution. Trends Ecol Evol 21:569-575.

Ezewudo MN, Joseph SJ, Castillo-Ramirez S, Dean D, Del Rio C, Didelot X, Dillon JA, Selden RF, Shafer WM, Turingan RS, Unemo M, and Read TD. 2015. Population structure of Neisseria gonorrhoeae based on whole genome data and its relationship with antibiotic resistance. PeerJ 3:e806. 10.7717/peerj.806

Fay J, Wycoff GJ, and Wu C-I. 2001. Positive and negative selection on the human genome. Genetics 158:1227-1234.

Feil EJ, Holmes EC, Bessen DE, Chan MS, Day NP, Enright MC, Goldstein R, Hood DW, Kalia A, Moore CE, Zhou J, and Spratt BG. 2001. Recombination within natural populations of pathogenic bacteria: short-term empirical estimates and long-term phylogenetic consequences. Proc Natl Acad Sci U S A 98:182-187. 10.1073/pnas.98.1.182

Peer) reviewing PDF | (2018:11:32791:2:1:NEW 23 May 2019) 
582

583

584

585

586

587

588

589

590

591

592

593

594

595

596

597

598

599

600

601

602

603

604

605

606

607

608

609

610

611

612

613

614

615

616

617

618

619

620

621

622

623

624

625
Galtier N. 2016. Adaptive Protein Evolution in Animals and the Effective Population Size Hypothesis. PLoS Genet 12:e1005774. 10.1371/journal.pgen.1005774

Gojobori J, Tang H, Akey JM, and $\mathrm{Wu} \mathrm{Cl}$. 2007. Adaptive evolution in humans revealed by the negative correlation between polymorphism and fixation pahses of evolution. Proc Natl Acad Sci USA 104:3907-3912.

Gordo I, and Dionisio F. 2005. Nonequilibrium model for estimating parameters of deleterious mutations. Phys Rev E Stat Nonlin Soft Matter Phys 71:031907. 10.1103/PhysRevE.71.031907

Gossmann TI, Woolfit M, and Eyre-Walker A. 2011. Quantifying the variation in the effective population size within a genome. Genetics 189:1389-1402. 10.1534/genetics.111.132654

Grad YH, Harris SR, Kirkcaldy RD, Green AG, Marks DS, Bentley SD, Trees D, and Lipsitch M. 2016. Genomic Epidemiology of Gonococcal Resistance to Extended-Spectrum Cephalosporins, Macrolides, and Fluoroquinolones in the United States, 2000-2013. Journal of Infectious Diseases 214:1579-1587. 10.1093/infdis/jiw420

Hao W, Ma JH, Warren K, Tsang RS, Low DE, Jamieson FB, and Alexander DC. 2011. Extensive genomic variation within clonal complexes of Neisseria meningitidis. Genome Biol Evol 3:1406-1418. 10.1093/gbe/evr119

Hill WG, and Robertson A. 1968. Linkage disequilibrium in finite populations. Theoret Appl Genet 38:226-231.

James J, Castellano D, and Eyre-Walker A. 2017. DNA sequence diversity and the efficiency of natural selection in animal mitochondrial DNA. Heredity (Edinb) 118:88-95. 10.1038/hdy.2016.108

Joseph B, Schneiker-Bekel S, Schramm-Gluck A, Blom J, Claus H, Linke B, Schwarz RF, Becker A, Goesmann A, Frosch M, and Schoen C. 2010. Comparative genome biology of a serogroup $B$ carriage and disease strain supports a polygenic nature of meningococcal virulence. J Bacteriol 192:5363-5377. 10.1128/JB.00883-10

Joseph B, Schwarz RF, Linke B, Blom J, Becker A, Claus H, Goesmann A, Frosch M, Muller T, Vogel U, and Schoen C. 2011. Virulence Evolution of the Human Pathogen Neisseria meningitidis by Recombination in the Core and Accessory Genome. PLOS ONE 6. ARTN e18441

10.1371/journal.pone.0018441

Kong Y, Ma JH, Warren K, Tsang RS, Low DE, Jamieson FB, Alexander DC, and Hao W. 2013. Homologous recombination drives both sequence diversity and gene content variation in Neisseria meningitidis. Genome Biol Evol 5:1611-1627. 10.1093/gbe/evt116

Lee RS, Seemann T, Heffernan H, Kwong JC, da Silva AG, Carter GP, Woodhouse R, Dyet KH, Bulach DM, Stinear TP, Howden BP, and Williamson DA. 2018. Genomic epidemiology and antimicrobial resistance of Neisseria gonorrhoeae in New Zealand. Journal of Antimicrobial Chemotherapy 73:353-364. 10.1093/jac/dkx405

Leffler EM, Bullaughey K, Matute DR, Meyer WK, Segurel L, Venkat A, Andolfatto P, and Przeworski M. 2012. Revisiting an old riddle: what determines genetic diversity levels within species? PLoS biology 10:e1001388. 10.1371/journal.pbio.1001388

Lewontin RC. 1974. The genetic basis of evolutionary change. New York: Columbia University Press.

Peer) reviewing PDF | (2018:11:32791:2:1:NEW 23 May 2019) 
626

627

628

629

630

631

632

633

634

635

636

637

638

639

640

641

642

643

644

645

646

647

648

649

650

651

652

653

654

655

656

657

658

659

660

661

662

663

664

665

666

667

668

Maddamsetti R, Hatcher PJ, Cruveiller S, Medigue C, Barrick JE, and Lenski RE. 2015. Synonymous Genetic Variation in Natural Isolates of Escherichia coli Does Not Predict Where Synonymous Substitutions Occur in a Long-Term Experiment. Mol Biol Evol 32:2897-2904. 10.1093/molbev/msv161

Martincorena I, and Luscombe NM. 2013. Non-random mutation: The evolution of targeted hypermutation and hypomutation. BioEssays : news and reviews in molecular, cellular and developmental biology 35:123-130. 10.1002/bies.201200150

Martincorena I, Seshasayee AS, and Luscombe NM. 2012. Evidence of non-random mutation rates suggests an evolutionary risk management strategy. Nature 485:95-98. 10.1038/nature10995

Maynard Smith J, and Haigh J. 1974. The hitch-hiking effect of a favourable gene. Genet Res 23:23-35.

McDonald JH, and Kreitman M. 1991. Adaptive evolution at the Adh locus in Drosophila. Nature 351:652-654.

Murray GGR, Soares AER, Novak BJ, Schaefer NK, Cahill JA, Baker AJ, Demboski JR, Doll A, Da Fonseca RR, Fulton TL, Gilbert MTP, Heintzman PD, Letts B, McIntosh G, O'Connell BL, Peck M, Pipes ML, Rice ES, Santos KM, Sohrweide AG, Vohr SH, Corbett-Detig RB, Green RE, and Shapiro B. 2017. Natural selection shaped the rise and fall of passenger pigeon genomic diversity. Science 358:951-954. 10.1126/science.aao0960

Ohta T. 1972. Population size and rate of evolution. J Mol Evol 1:305-314.

Ohta T. 1977. Extension of the neutral mutation drift hypothesis. In: Kimura M, ed. Molecular Evolution and Polymorphism. Mishima: National Institute of Genetics, 148-167.

Ohta T. 1992. The nearly neutral theory of molecular evolution. Ann Rev Ecol Syst 23:263-286.

Parkhill J, Achtman M, James KD, Bentley SD, Churcher C, Klee SR, Morelli G, Basham D, Brown D, Chillingworth T, Davies RM, Davis P, Devlin K, Feltwell T, Hamlin N, Holroyd S, Jagels K, Leather S, Moule S, Mungall K, Quail MA, Rajandream MA, Rutherford KM, Simmonds M, Skelton J, Whitehead S, Spratt BG, and Barrell BG. 2000. Complete DNA sequence of a serogroup A strain of Neisseria meningitidis Z2491. Nature 404:502-506. $10.1038 / 35006655$

Peng J, Yang L, Yang F, Yang J, Yan Y, Nie H, Zhang X, Xiong Z, Jiang Y, Cheng F, Xu X, Chen S, Sun L, Li W, Shen Y, Shao Z, Liang X, Xu J, and Jin Q. 2008. Characterization of ST-4821 complex, a unique Neisseria meningitidis clone. Genomics 91:78-87. 10.1016/j.ygeno.2007.10.004

Roberts L. 2008. An ill wind, bringing meningitis. Science 320:1710-1715.

Romiguier J, Gayral P, Ballenghien M, Bernard A, Cahais V, Chenuil A, Chiari Y, Dernat R, Duret L, Faivre N, Loire E, Lourenco JM, Nabholz B, Roux C, Tsagkogeorga G, Weber AAT, Weinert LA, Belkhir K, Bierne N, Glemin S, and Galtier N. 2014. Comparative population genomics in animals uncovers the determinants of genetic diversity. Nature 515:261-U243. $10.1038 /$ nature13685

Rusniok C, Vallenet D, Floquet S, Ewles H, Mouze-Soulama C, Brown D, Lajus A, Buchrieser C, Medigue C, Glaser P, and Pelicic V. 2009. NeMeSys: a biological resource for narrowing the gap between sequence and function in the human pathogen Neisseria meningitidis. Genome Biol 10:R110. 10.1186/gb-2009-10-10-r110

PeerJ reviewing PDF | (2018:11:32791:2:1:NEW 23 May 2019) 
669

670

671

672

673

674

675

676

677

678

679

680

681

682

683

684

685

686

687

688

689

690

691

692

693

694

695

696

697

698

699

700

701

702

703

704

705

706

707

708

709

710

Sattath S, Elyashiv E, Kolodny O, Rinott Y, and Sella G. 2011. Pervasive Adaptive Protein Evolution Apparent in Diversity Patterns around Amino Acid Substitutions in Drosophila simulans. PLoS Genetics 7. ARTN e1001302

10.1371/journal.pgen.1001302

Schoen C, Blom J, Claus H, Schramm-Gluck A, Brandt P, Muller T, Goesmann A, Joseph B, Konietzny S, Kurzai O, Schmitt C, Friedrich T, Linke B, Vogel U, and Frosch M. 2008. Whole-genome comparison of disease and carriage strains provides insights into virulence evolution in Neisseria meningitidis. Proc Natl Acad Sci U S A 105:3473-3478. $10.1073 /$ pnas. 0800151105

Schoen C, Weber-Lehmann J, Blom J, Joseph B, Goesmann A, Strittmatter A, and Frosch M. 2011. Whole-genome sequence of the transformable Neisseria meningitidis serogroup $A$ strain WUE2594. J Bacteriol 193:2064-2065. 10.1128/JB.00084-11

Spratt BG. 1988. Hybrid penicillin-binding proteins in penicillin-resistant strains of Neisseria gonorrhoeae. Nature 332:173-176. 10.1038/332173a0

Spratt BG, Zhang QY, Jones DM, Hutchison A, Brannigan JA, and Dowson CG. 1989. Recruitment of a penicillin-binding protein gene from Neisseria flavescens during the emergence of penicillin resistance in Neisseria meningitidis. Proc Natl Acad Sci U S A 86:8988-8992.

Tajima F. 1989. Statistical method for testing the neutral mutation hypothesis by DNA polymorphism. Genetics 123:585-595.

Tettelin H, Saunders NJ, Heidelberg J, Jeffries AC, Nelson KE, Eisen JA, Ketchum KA, Hood DW, Peden JF, Dodson RJ, Nelson WC, Gwinn ML, DeBoy R, Peterson JD, Hickey EK, Haft DH, Salzberg SL, White O, Fleischmann RD, Dougherty BA, Mason T, Ciecko A, Parksey DS, Blair E, Cittone H, Clark EB, Cotton MD, Utterback TR, Khouri H, Qin H, Vamathevan J, Gill J, Scarlato V, Masignani V, Pizza M, Grandi G, Sun L, Smith HO, Fraser CM, Moxon ER, Rappuoli R, and Venter JC. 2000. Complete genome sequence of Neisseria meningitidis serogroup B strain MC58. Science 287:1809-1815.

Vazquez JA, Delafuente L, Berron S, Orourke M, Smith NH, Zhou JJ, and Spratt BG. 1993. Ecological Separation and Genetic Isolation of Neisseria-Gonorrhoeae and NeisseriaMeningitidis. Current Biology 3:567-572. Doi 10.1016/0960-9822(93)90001-5

Vos M, and Didelot X. 2009. A comparison of homologous recombination rates in bacteria and archaea. ISME J 3:199-208. ismej200893 [pii]

10.1038/ismej.2008.93

Welch JJ, Eyre-Walker A, and Waxman D. 2008. Divergence and Polymorphism Under the Nearly Neutral Theory of Molecular Evolution. J Mol Evol 67:418-426. 10.1007/s00239008-9146-9

WHO. 2012. Global action plan to control the spread and impact of antimicrobial resistance in Neisseria gonorrhoeae.

Yazdankhah SP, Kriz P, Tzanakaki G, Kremastinou J, Kalmusova J, Musilek M, Alvestad T, Jolley KA, Wilson DJ, McCarthy ND, Caugant DA, and Maiden MC. 2004. Distribution of serogroups and genotypes among disease-associated and carried isolates of Neisseria meningitidis from the Czech Republic, Greece, and Norway. J Clin Microbiol 42:51465153. 10.1128/JCM.42.11.5146-5153.2004

Peer) reviewing PDF | (2018:11:32791:2:1:NEW 23 May 2019) 
711 Yu D, Jin Y, Yin ZQ, Ren HG, Zhou W, Liang L, and Yue JJ. 2014. A Genome-Wide Identification of

712 Genes Undergoing Recombination and Positive Selection in Neisseria. Biomed Research

713 International. Artn 815672

714 10.1155/2014/815672

715 Zhang Y, Yang J, Xu L, Zhu Y, Liu B, Shao Z, Zhang X, and Jin Q. 2014. Complete Genome

716

717 Sequence of Neisseria meningitidis Serogroup A Strain NMA510612, Isolated from a

718 Patient with Bacterial Meningitis in China. Genome Announc 2.

719 10.1128/genomeA.00360-14

720 


\section{Table 1 (on next page)}

Nucleotide diversity estimates across all sites in the core genome (pi) and at 0-fold nonsynonymous sites $\left(\mathrm{pi}_{\mathrm{N}}\right)$ and 4 -fold synonymous sites $\left(\mathrm{pi}_{\mathrm{S}}\right)$. 
1

\begin{tabular}{|l|l|l|l|}
\hline & $\pi$ & $\pi_{\mathrm{S}}$ & $\pi_{\mathrm{N}}$ \\
\hline N. gonorrhoeae & $0.0029(0.0008)$ & $0.007(0.002)$ & $0.0014(0.0004)$ \\
\hline N. meningitidis & $0.022(0.007)$ & $0.06(0.02)$ & $0.007(0.002)$ \\
\hline Ratio & 7.6 & 8.6 & 5.0 \\
\hline
\end{tabular}

2

3

4 Table 1. Diversity estimates across all sites in the core genome $(\pi)$ and at 0 -fold non-

5 synonymous sites $\left(\pi_{N}\right)$ and 4-fold synonymous sites $\left(\pi_{\mathrm{S}}\right)$.

6 


\section{Table 2 (on next page)}

Recombination rate estimates obtained from ClonalFrameML along with their $95 \%$ confidence intervals.

Given is the rate at which recombination tracts initiate $(R)$ relative to the rate of mutation (theta), both multiplied by the effective population size, the average length of recombination tracts (delta) and the proportion of sites that differ to the resident sequence (mu), along with the rate at which sites change due to recombination relative to mutation $(\mathrm{r} / \mathrm{m})$ 
1

\begin{tabular}{|l|l|l|l|l|l|l|}
\hline Species & $\mathrm{R} / \theta$ & $\delta$ & $v(\%)$ & $\mathrm{r} / \mathrm{m}$ & $\theta\left(\times 10^{-3}\right)$ & $\mathrm{R}\left(\times 10^{-4}\right)$ \\
\hline $\mathrm{Ng}$ & $\begin{array}{l}0.41(0.39, \\
0.43)\end{array}$ & $70(67,72)$ & $\begin{array}{l}6.9(6.8, \\
7.1)\end{array}$ & $\begin{array}{l}2.0(1.8, \\
2.2)\end{array}$ & $1.0(0.8,1.2)$ & $4.0(3.0,5.0)$ \\
\hline $\mathrm{Nm}$ & $\begin{array}{l}1.2(1.2, \\
1.3)\end{array}$ & $\begin{array}{l}99(98, \\
100)\end{array}$ & $\begin{array}{l}5.3(5.3, \\
5.4)\end{array}$ & $\begin{array}{l}6.4(6.2, \\
6.7)\end{array}$ & $3.0(2.5,3.5)$ & $36(30,44)$ \\
\hline $\begin{array}{l}\text { Ratio } \\
(\mathrm{Nm} / \mathrm{Ng})\end{array}$ & 3.0 & 1.4 & 0.77 & 3.2 & 3.0 & 9.3 \\
\hline
\end{tabular}

2

3

4 Table 2. Recombination rate estimates obtained from ClonalFrameML along with their 95\% 5 confidence intervals.

6 
Figure 1 (on next page)

Decay in linkage disequilibrium with the distance between sites.

Linkage disequilibrium, as measured by $r^{2}$ (Hill \& Robertson 1968), between pairs of polymorphic sites as a function of the distance between sites for (A) all sites and (B) for those sites not inferred to have undergone hLGT. Each point represents the average $r^{2}$ between all pairs of points separated by a certain distance in bins of $10 \mathrm{bp}$ between 0 and $100 \mathrm{bp}$, a bin of 101 to $200 \mathrm{bp}$ and then bins of $200 \mathrm{bp}$ upto $800 \mathrm{bp}$. $\mathrm{Nm}$ in green, $\mathrm{Ng}$ in red. 
PeerJ

A 0.8

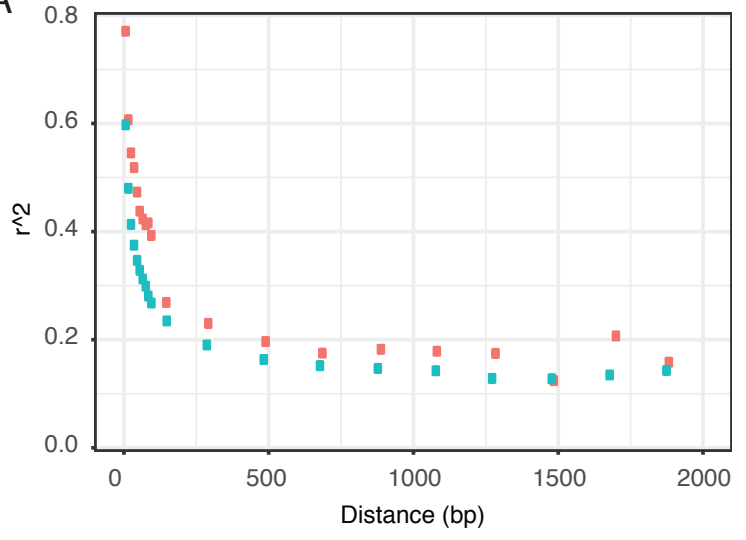

Manuscript to be reviewed

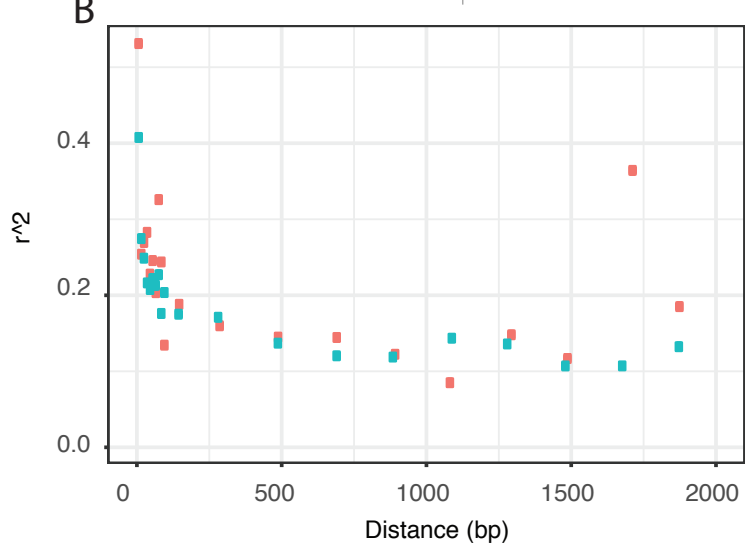


Figure 2 (on next page)

Correlation between non-synonymous and synonymous diversity across the genome.

he correlation between the log of the non-synonymous nucleotide diversity and the log of the synonymous diversity for core genes in A) Nm and B) Ng. Points in green are genes with evidence of hLGT and red are those genes without evidence of hLGT. Note that some genes are excluded because they have either no non-synonymous or synonymous diversity. 

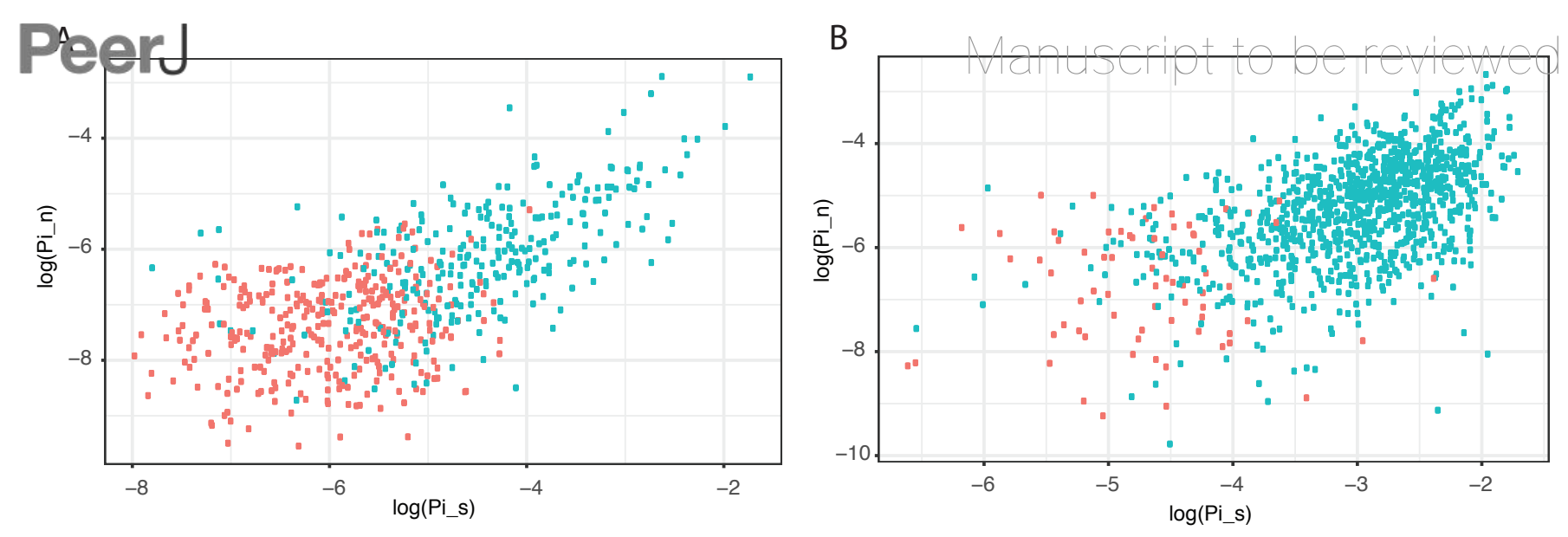
Figure 3 (on next page)

Correlation between non-synonymous and synonymous diversity excluding regions with evidence of hLGT.

he correlation between the log of the non-synonymous nucleotide diversity plotted and the log of the synonymous diversity for regions of the genome that have not undergone hLGT. Green is Nm, red is Ng. Also shown are the lines of best fit. 


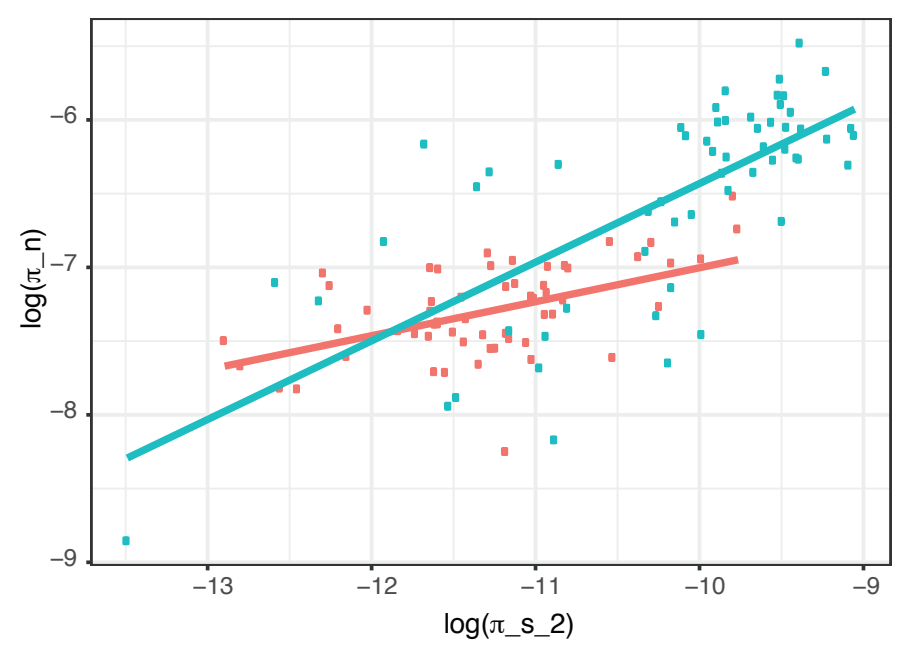


Figure 4 (on next page)

Synonymous diversity around sites fixed for either non-synonymous or synonymous substitutions.

Average synonymous diversity in A) $\mathrm{Nm}$ and B) $\mathrm{Ng}$ around sites that are fixed for either a non-synonymous (red) or synonymous (green) substitution between $\mathrm{Nm}$ and $\mathrm{Ng}$. 

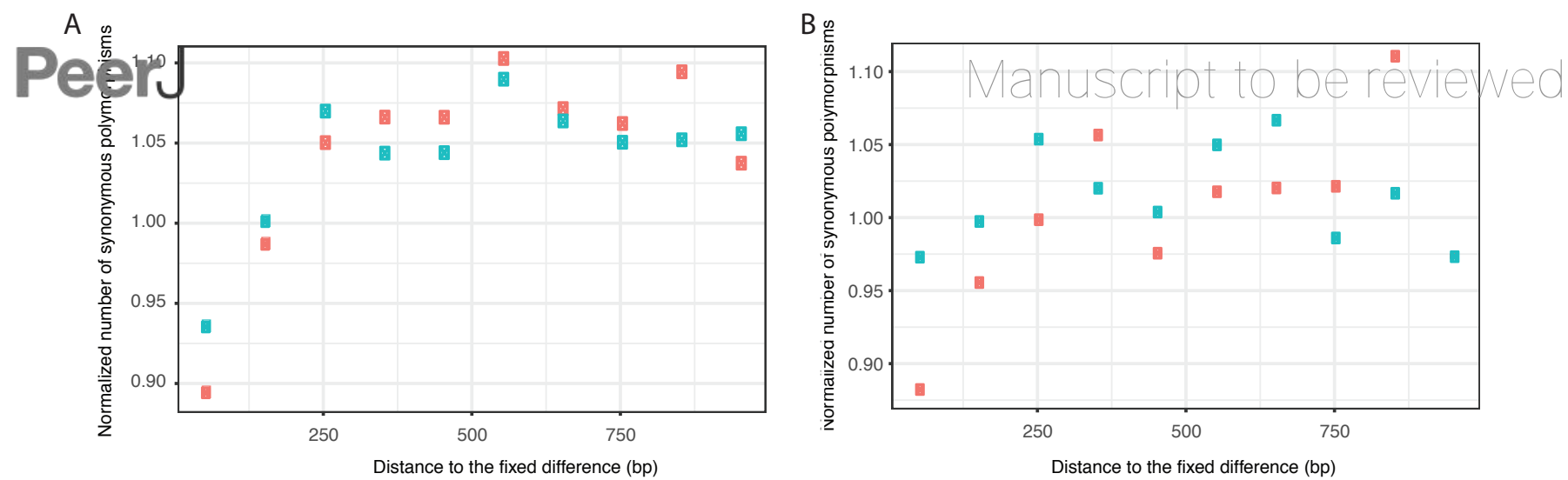
Figure 5 (on next page)

hLGT tracts at the start and end of genes,

The number of sequences inferred to be due to hLGT in both species as a function of the

distance from the A) start and B) end of genes, where the distance was the proportion of the gene length from the start and end 

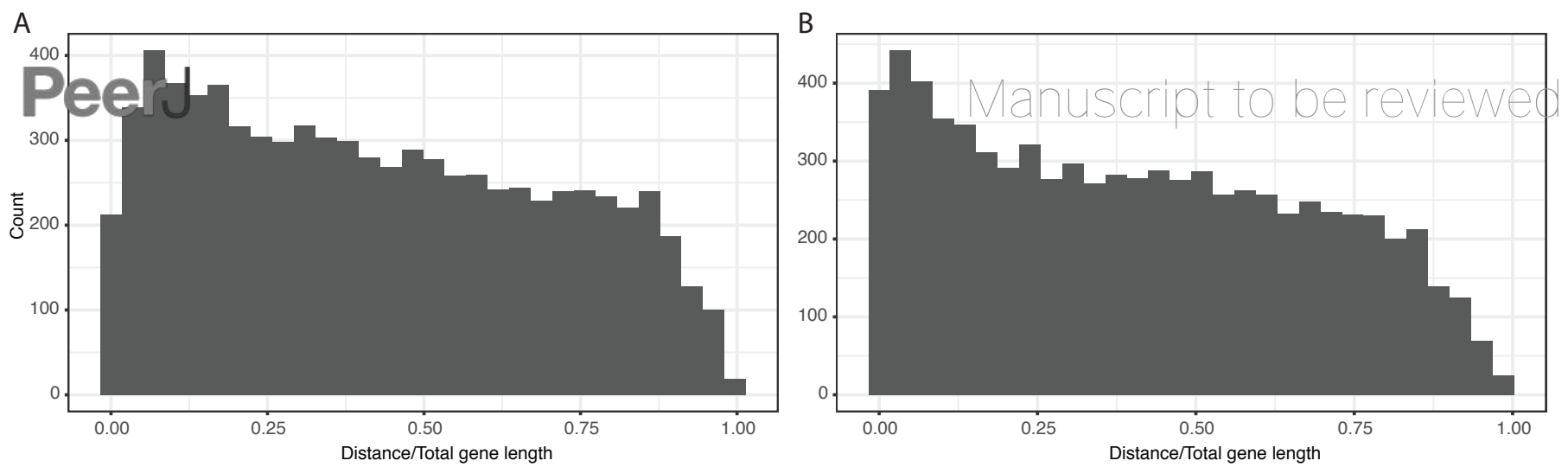